\title{
AD-1578 - Calcium-phosphorus product is associated with non-alcoholic fatty liver disease in type 2 diabetes mellitus
}

Xin Wen, Xianghai Zhou, Xiantong Zou, Lihong Gao, Fuhong Zhang, Da Chen, Meimei Guo Department of Endocrinology and Metabolism , Peking University People's Hospital

Table 1-Clinical characteristics of participants by nonalcoholic fatty liver disease (NAFLD) category

\begin{tabular}{lccc}
\hline \multicolumn{1}{c}{ Variables } & non-NAFLD & NAFLD & $\mathrm{P}$ \\
\hline Male(\%) & $356(51)$ & $393(53.6)$ & 0.309 \\
Age(years) & $58.9 \pm 12.5$ & $54.4 \pm 13.6$ & $<0.001$ \\
Duration(years) & $11.0(6.0-18.0)$ & $8.3(3.0-14.0)$ & $<0.001$ \\
$\mathrm{BMI}\left(\mathrm{kg} / \mathrm{m}^{2}\right)$ & $23.85 \pm 3.16$ & $27.23 \pm 3.81$ & $<0.001$ \\
$\mathrm{HbA}_{1 \mathrm{c}}(\%)$ & $8.9(7.4-10.7)$ & $9.2(7.8-10.7)$ & 0.007 \\
Fasting glucose(mmol/L) & $6.91(5.58-8.83)$ & $7.37(6.01-9.40)$ & 0.001 \\
Triglyceride(mmol/L) & $1.22(0.91-1.64)$ & $1.86(1.36-2.60)$ & $<0.001$ \\
HDL-c(mmol/L) & $1.04(0.88-1.26)$ & $0.92(0.80-1.06)$ & $<0.001$ \\
Uric acid(umol/L) & $305 \pm 79$ & $351 \pm 93$ & $<0.001$ \\
Serum calcium(mmol/L) & $2.20 \pm 0.13$ & $2.23 \pm 0.13$ & $<0.001$ \\
Serum phosphorus(mmol/L) & $1.24 \pm 0.19$ & $1.28 \pm 0.18$ & $<0.001$ \\
Calcium-phosphorus product $\left(\mathrm{mg}^{2} / \mathrm{dl}^{2}\right.$ ) & $33.81 \pm 5.88$ & $35.25 \pm 5.70$ & $<0.001$ \\
PTH(IU/mL) & $31.82(24.13-39.57)$ & $32.30(25.10-41.27)$ & 0.056 \\
Vitamin D(nmol/L) & $39.70(30.72-51.42)$ & $37.77(30.27-48.32)$ & 0.058 \\
\hline
\end{tabular}

Continuous variables are expressed as mean \pm standard division and compared by $t$ test. Categorical variables were expressed by median (interquartile range) and compared by $\mathrm{x} 2$ test. $\mathrm{BMI}=$ body mass index; HDL$\mathrm{C}=$ high density lipoprotein cholesterol; $\mathrm{PTH}=$ parathyroid hormone.

\section{Objective}

To determine whether calcium and phosphorus levels are associated with the prevalence of non-alcoholic fatty liver disease ( NAFLD ) in patients of type 2 diabetes mellitus (T2DM) in China.

\section{Method}

2715 With T2DM who were admitted to the ward of the department of
Peking University People's Hospital were enrolled

\section{Results}

\section{Clinical characteristics}

The prevalence of NAFLD is 52.2\%(1805) in T2DM patients. Mean age of the participants was 56.2 \pm 13.3 years. Compared with the group without NAFLD, individuals diagnosed with NAFLD were younger, had shorter duration
Model 1

P for trend

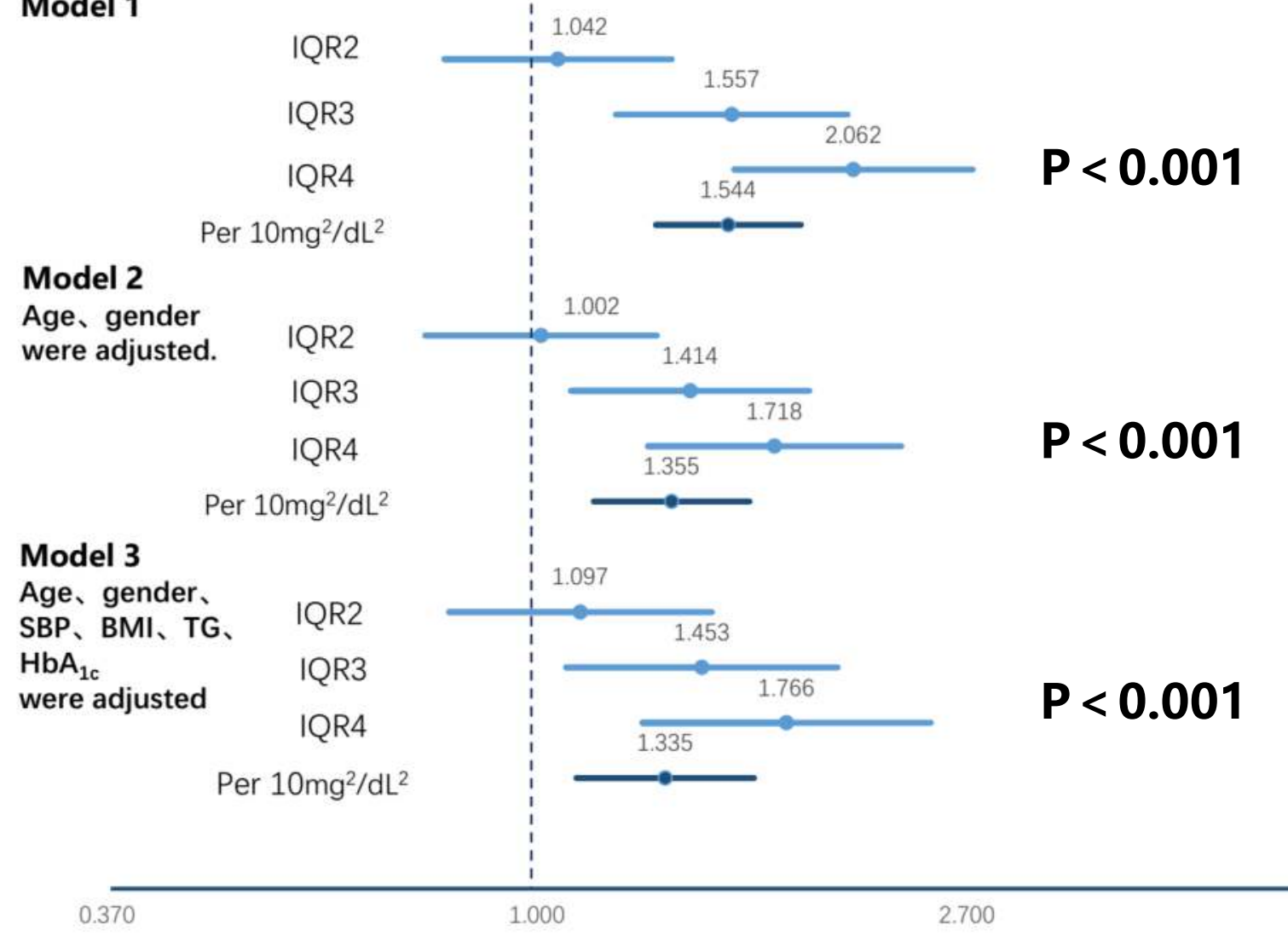

Figure 1-Association between calcium-phosphorus product and presence of non-alcoholic fatty liver disease using logistic regression.

$\mathrm{SBP}=$ systolic blood pressure $; \mathrm{BMI}=$ body mass index $; \mathrm{TG}=$ triglyceride.

of diabetes. Individuals with NAFLD have higher levels of $\mathrm{BMI}, \mathrm{HbA1c}$, fasting glucose, triglyceride and uric acid. Serum calcium, phosphorus and calcium-phosphorus product were higher in NAFLD group compared with the group without NAFLD (Table 1).

2 Association between calcium-phosphorus product and non-alcoholic fatty liver

The prevalence of NAFLD increased with increasing calcium-phosphorus product quartiles. In multiple logistic regression model, the ORs for the prevalence of NAFLD were increasing in IQR2 group, IQR3 group and IQR4 group in reference to IQR1 group, after adjusting sex, age, SBP, BMI, HbA1c and TG (Figure 1).

\section{Confounding factor of vitamin D and PTH}

Similar results were found after vitamin D and PTH was adjusted in 1202 participants with data on vitamin D and PTH.

\section{Conclusion}

Increasing level of calcium-phosphorus product is associated with the presence of NAFLD in this Chinese hospitalized T2DM population.

\section{Limitation}

This cross-sectional study cannot prove a causal relationship between calcium-phosphorus product and NAFLD. Potential unmeasured confounding factors may be not excluded even though many possibilities were taken into consideration. 